\title{
OPENING TALK
}

\section{Two Sides to Every Story - including cotransport mechanism.}

\author{
Richard J Naftalin
}

Cardiovascular Division, School of Medicine, King's College London, Waterloo Campus, Stamford St. London, SE1 9NH.

\section{Introduction}

Cotransport is recognised as being highly asymmetric. Cotransporters have lower affinities for organic ligands at their endofacial (cytosolic) than at the exofacial surface. This property complements the requirement that they generate high intracellular concentrations of cotransported ligands, e.g. neurotransmitter cotransporters have inside affinities for noradrenalin, serotonin and dopamine four or five orders lower than at the outside and accumulate these neurotransmitters within the cytosol to concentrations 4-5 orders higher than present within the synaptic cleft (1). Epithelial cotransporters too have asymmetric affinities towards their organic ligands.

\section{Asymmetry and selectivity of SGLT1 and PEPT1 cotransport.}

Glucose-dependent electrogenic $\mathrm{Na}^{+}$currents can be monitored in either excised outside-out or insideout patches of Xenopus oocyte membranes overexpressing the sodium glucose cotransporter SGLT1. The apparent affinities for $\mathrm{Na}^{+}$are symmetrical, the $\mathrm{K}_{\mathrm{m}}$ for $\mathrm{Na}^{+}$is $40-50 \mathrm{mM}$ on both sides (2-4). However, the affinities for glucose (Glc) or $\alpha$-methyl glucoside $(\alpha M G)$ are very asymmetric.

It is generally assumed that transporter asymmetry arises from the conformational changes occurring during inversion of the central binding site between outward to inward facing postures. This rearrangement is thought to alter the ligand-protein interactions determining affinity and selectivity. This view is supported by the finding that the selectivity of SGLT1 of the outward face towards a range of transported sugars differs from that of the inward face (3). The affinity ratio between the internal and external sites of well transported sugars, like $\alpha \mathrm{MG}$, glucose and galactose, is much higher than for a less well transported sugar like 3-O methyl glucose (3-OMG) $(2,3)$. This might suggest that the endofacial site has different bonding groups with ligand than the exofacial site as a result of the rearrangement occurring during site inversion. Similar changes in selectivity for various glycine dipeptides occur with PEPT1 (5).

\section{Constraints on alternating access cotransporter kinetics.}

The alternating access model for cotransport entails some key kinetic constraints. The first is that the product of all the rates in the clockwise direction around all possible cycles within a network is equal 
to the product of the rates in the anticlockwise direction (figure 1). This is a consequence of the necessity that that all the flows of matter and energy must be zero at equilibrium. Compensation within the network between one or more energy cycles for a shortfall in another is impermissible (6). Any implicit internally derived energy source produced from asymmetric rates of vacant carrier movements is simply erroneous. An asymmetric ratio of vacant carrier rates implies that energy is produced by this movement. Thus when is $\mathrm{k}_{\mathrm{oi}} \neq \mathrm{k}_{\mathrm{io}}$, then the Gibbs free energy $\Delta \mathrm{G}=-\operatorname{RTLN}\left(\mathrm{k}_{\mathrm{oi} /} \mathrm{k}_{\mathrm{io}}\right)$ of the process $\neq 0$ ( $\mathrm{k}_{\mathrm{oi}}$ is the rate of movement from outside to inside and $\mathrm{k}_{\mathrm{io}}$ is the reverse rate from inside to outside). This implies that all membrane spanning rate processes are symmetrical (6).

\section{Problems relating to the Eskandari et al cotransport model for SGLT1}

\section{Asymmetric transmembrane processes.}

The well-received cotransport model proposed by Eskandari et al (3) has two asymmetric membrane spanning processes (figure 1). The ratio of rates of vacant carrier movement $\mathrm{k}_{\mathrm{Coi}} / \mathrm{k}_{\mathrm{Cio}}=350 / 3=117$ is equivalent to $\Delta \mathrm{G}=$ $12.25 \mathrm{~kJ} /$ mole and the ratio of the rates of $\mathrm{Na}^{+}$ leakage via the $\mathrm{Na}_{2} \mathrm{C}$ path $0.3 / 9.1 \times 10^{-4}=330$ equivalent to $\Delta \mathrm{G}=-14.9 \mathrm{~kJ} / \mathrm{mole}$. This energy is supposed to compensate for the difference in binding energy affinities of $\mathrm{Na}^{+}$and sugar ligands between the outward and inward facing sites of the transporter. The rate
Rate constants and affinities for SGLT1 (Eskandari et al. 2005)

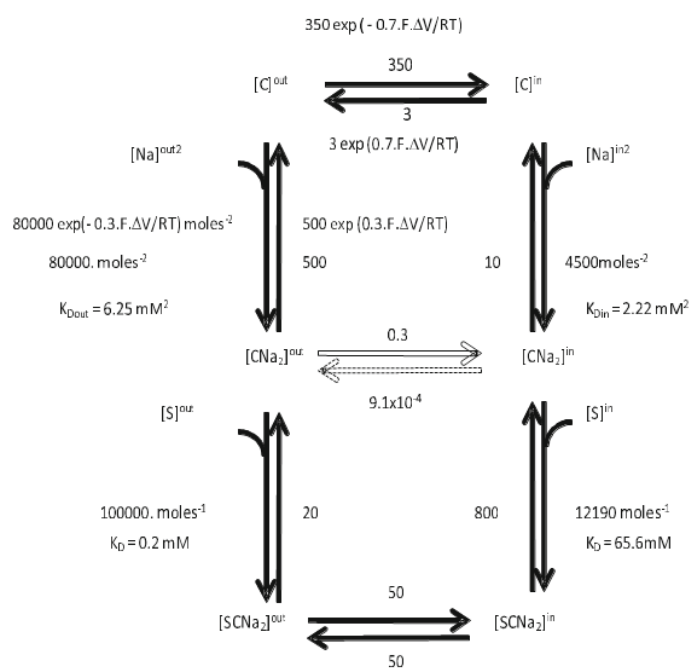
constants for vacant carrier and $\mathrm{Na}^{+}$leak spanning the membrane are independent of sugar; the only transmembrane rate process involving sugar is the canonical cotransport process itself, that of the carrier complexed with $\mathrm{Na}^{+}$and sugar, $\mathrm{Na}_{2} \mathrm{SC}$. This latter process is considered to be symmetrical $\mathrm{k}_{\mathrm{Na} 2 \mathrm{SCoi}}=\mathrm{k}_{\mathrm{Na} 2 \mathrm{SCio}}=50$ (figure 1).

\section{Asymmetric selectivity problem.}

The Eskandari model imposes another constraint in that the sugar affinities ratio between the outward and inward facing sites must be the same for all sugars. As the non sugar transporting components of the model are independent for all sugars; Detailed Balance imposes a rigid framework. The lower cycle in figure 1 requires that the ratios of sugar affinities $\left(\mathrm{K}_{\mathrm{din}} / \mathrm{K}_{\mathrm{do}}\right)$ between the external and internal faces of all sugars should be the same as the fixed ratio of $\left\{\left(\mathrm{k}_{\mathrm{Na} 2 \mathrm{Coi}} / \mathrm{k}_{\mathrm{Na} 2 \mathrm{cio}}\right) /\left(\mathrm{k}_{\mathrm{Na} 2 \mathrm{SCoi}_{\mathrm{i}}} / \mathrm{k}_{\mathrm{Na} 2 \mathrm{Scio}}\right)\right\}=329 / 1$. Consequently the model predicts that no difference in sugar selectivity should exist between the two sides. The inside/ outside affinity ratio of all sugars should be $65.6 / 0.2=329$. The affinity ratios for the entire cycle are $\left\{\left(\mathrm{K}_{\mathrm{dNa} 2 i n} \cdot \mathrm{K}_{\mathrm{dNa} 2 \mathrm{Sin}}\right) /\left(\mathrm{K}_{\mathrm{dNa} 2 \text { out }} \cdot \mathrm{K}_{\mathrm{dNa} 2 \mathrm{Sout}}\right)\right\}=\{(2.22 \times 65.6) /(6.25 \times 0.2)\}=117$. 
Eskandari et al (3) report that the apparent affinity ratios of $\alpha \mathrm{MG}$ galactose and Glc $\cong 100$ which agree with the model prediction, but the affinity ratio of $3 \mathrm{OMG} \cong 1.0$ fits very poorly.

\section{An alternative model for cotransport.}

Since there are inconsistencies with the alternating access cotransport model, another mechanism for cotransport should be considered. Uphill sugar transport can be driven by frictional collisions with $\mathrm{Na}^{+}$ions during their downhill trajectory through SGLT1 (6) (figure 2). In narrow localized regions in channel within the transporter protein where $\mathrm{Na}^{+}$ions and sugar share a common pathway, collision and exchange energy with a force related to the closeness of ligands contact may occur: the closer the contact, the greater the efficiency of energy exchanged. Energy contained within the downhill $\mathrm{Na}^{+}$gradient can be conferred upon glucose to induce its Friction coupled model of $\mathrm{Na}$ and glucose transport with osmotic water flow uphill flow. Independent ligand leakage is

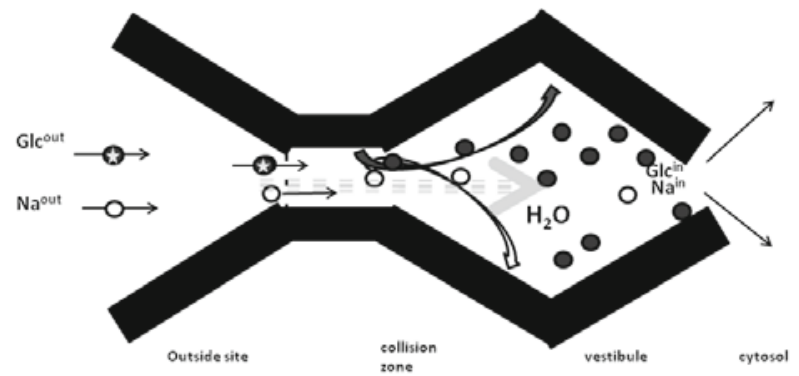
intrinsic to this model.

The model makes a number of predictions that differ from the alternate access model. Here are a few:-

- A sugar with weak frictional interactions with $\mathrm{Na}^{+}$e.g. 3-OMG will generate less cotransport electrogenic current and be accumulated to a lesser within the cytosol. Raised cytosolic $\mathrm{Na}^{+}$ will generate a smaller increase in unidirectional 3-OMG exit rates than sugars with higher frictional interactions e.g. $\alpha M G$ or Glc whose exit is retarded more by the $\mathrm{Na}^{+}$gradient. These phenomena have all been demonstrated with the bidirectional fluxes of $\alpha \mathrm{MG}$ and 3OMG across the rabbit ileal brush border (7).

- Another prediction is that reducing the $\mathrm{Na}^{+}$gradient either by raising intracellular $\mathrm{Na}^{+}$or by reducing external $\mathrm{Na}^{+}$should reduce both net and unidirectional glucose influx- and increase unidirectional glucose efflux, as has been observed (7-9). The alternating access model predicts that raising intracellular $\mathrm{Na}^{+}$should have a negligible effect on unidirectional glucose influx and reduction of external $\mathrm{Na}^{+}$should have a negligible effect on glucose efflux.

Similar interactions occur with reverse flow of dipeptides across the $\mathrm{H}^{+}$-polypeptide cotransporter, PEPT1 (10).

\section{The frictional model in relation to known transporter protein crystal structures.}

1. In its reverse transport mode isolated oocyte membrane patches expressing the human dopamine transporter hDAT oscillates between slow and high frequency release of DA. These oscillations correlate with increases in ionic conductance (11). The transient release of DA 
together with increased conductance is consistent with widening of the narrow collision pathway. Faster DA release is consistent with to loss of the retarding force on exit caused by the inward directed collisions with $\mathrm{Na}^{+}$.

2. Progressive decreases of dipeptide (glycyl-glycine) induced currents are observed in isolated xenopus oocyte patches expressing PEPT1. These findings support the view that the transported solute accumulates 'close' to the membrane (4).

3. The phloridzin-sensitive water transport observed with SLGT1 expressed in xenopus oocytes following exposure to $\alpha \mathrm{MG}(12)$ is consistent with the view that water 'cotransport' is caused by osmotic pressure generated by solute accumulation within a cavity inside the transport pathway of $\operatorname{SGLT1}(12,13)$. Support for this view is the very rapid onset of the water flow after exposure to $\alpha \mathrm{MG}$. This rise time in water cotransport is too rapid to be generated by solute accumulation within a cytosolic unstirred layer.

Cavities within the transport pathway permitting transported solute accumulation account for several other phenomena, the transporter-channel modulation (11); the relaxation of cotransport currents and water cotransport (5). This frictional model of cotransport can easily be projected onto known transporter structures without any conjectural conformation changes being required.

\section{References}

1) Robertson SD, Matthies HJ, Galli A (2009) A Closer Look At Amphetamine-Induced Reverse Transport And Trafficking Of The Dopamine And Norepinephrine Transporters. Mol Neurobiol 39:73-80

2) Sauer GA, Nagel G, Koepsell H, Bamberg E, Hartung K (2000) Voltage And Substrate Dependence Of The Inverse Transport Mode Of The Rabbit Na+/Glucose Cotransporter (SGLT1). FEBS Letters 469 98-100

3) Eskandari S, Wright EM, Loo DDF Kinetics Of The Reverse Mode Of The Na+/Glucose Cotransporter. (2005) J Membr Biol 204: 23-32

4) Parent L, Supplisson S, Loo DD, Wright EM (1992) Electrogenic properties of the cloned $\mathrm{Na}+$ /glucose cotransporter: I. Voltage-clamp studies. J Membr Biol 125:49-62

5) Renna MD Oyadeyi AS, Bossi E, Kottra G, Peres A (2010) Functional And Structural Determinants Of Reverse Operation In The pH-Dependent Oligopeptide Transporter Pept1 Cell Mol Life Sci DOI: $10.1007 / \mathrm{s} 00018-010-0604-3$

6) Naftalin RJ (2010) Reassessment Of Models Of Facilitated Transport And Cotransport. J Membr Biol 234 75-112

7) Holman GD, Naftalin RJ (1976) Transport Of 3-O-Methyl D-Glucose And Beta-Methyl DGlucoside By Rabbit Ileum. Biochim Biophys Acta 433: 597-614 
8) Semenza G, Kessler M, Hosang M, Weber J, Schmidt U (1984) Biochemistry Of The $\mathrm{Na}^{+}$, DGlucose Cotransporter Of The Small Intestinal Brush Border Membrane. (1984). Biochim Biophys Acta 779: 343-379

9) Centelles JJ, Kinne RKH, Heinz E (1991) Energetic Coupling Of Na-Glucose Cotransport. Biochim Biophys Acta 1095: 239-249

10) Kottra G, Frey I, Daniel H (2009) Inhibition Of Intracellular Dipeptide Hydrolysis Uncovers Large Outward Transport Currents Of The Peptide Transporter PEPT1 In Xenopus Oocytes. Pflugers Arch Eur J Physiol 457: 809-820

11) Kahlig KM, Binda F, Khoshbouei H, Blakely RD, McMahon DG, Javitch JA, Galli A. 2005 Amphetamine Induces Dopamine Efflux Through A Dopamine Transporter Channel. Proc Natl Acad Sci U S A 102:3495-3500.

12) Zeuthen T (2010) Water-Transporting Proteins. J Membr Biol. 234:57-73.

13) Naftalin RJ (2008) Osmotic Water Transport With Glucose In GLUT2 And SGLT. Biophys J 94:3912-3923 